\title{
Strengthening District Health Teams Capacity in Surveillance Systems and Response to Public Health Threats in Western Uganda through Field Epidemiology Training Program (FETP)
}

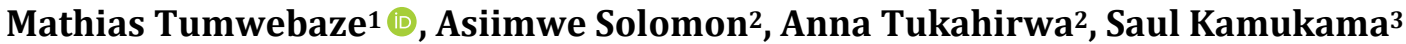 \\ ${ }^{1}$ Department of Public Health, Bishop Stuart University, Mbarara, Uganda \\ ${ }^{2}$ Kabarole District Local Government, District Health Office Fort Portal Town, Western Uganda \\ ${ }^{3}$ Makerere University College of Health Sciences, Kampala, Uganda \\ Email: mathiastumwebaze@gmail.com, sasiimwemedd@gmail.com, annatukahirwa@yahoo.co.uk, skamukama@musph.ac.ug
}

How to cite this paper: Tumwebaze, M., Solomon, A., Tukahirwa, A. and Kamukama, S. (2020) Strengthening District Health Teams Capacity in Surveillance Systems and Response to Public Health Threats in Western Uganda through Field Epidemiology Training Program (FETP). Open Journal of Epidemiology, 10, 132-145.

https://doi.org/10.4236/ojepi.2020.102012

Received: March 21, 2020

Accepted: May 4, 2020

Published: May 7, 2020

Copyright $\odot 2020$ by author(s) and Scientific Research Publishing Inc. This work is licensed under the Creative Commons Attribution International License (CC BY 4.0).

http://creativecommons.org/licenses/by/4.0/

\begin{abstract}
Aim: To strengthen the District Capacity in surveillance for effective detection, Reporting and Response to Public Health threats. Background: The overall aim of a good surveillance system is to strengthen the capacity of a Health system through training of health personnel who can conduct effective surveillance activities. A good surveillance system is achieved through improved use of complete and timely health information to detect changes in time to institute a rapid response to the suspected outbreak of Public Health events. This assessment followed a 3-month Field Epidemiology Training program undertaken by the investigator who applied the acquired knowledge and skills in completion of the assessment. Study Design: It was a descriptive cross-sectional, institutional based epidemiological investigation conducted at district level and Health Centre $1 \mathrm{~V}$ in Kabarole from $15^{\text {th }}$ December 2019-March 2020. eReports were retrieved from DHIS-2 for epidemiological weeks 44 in 2019 to week 3 in 2020. Data analysis: Micro soft word excel program was used to determine the reporting rates, epidemic disease trends and construction of malaria channel. SWOT analysis was done to identify poor HMIS reporting as the lead surveillance quality challenge and route cause analysis done to determine underlying causes. Results: Weekly reports analyzed were from a total of 53 Health facilities and one Health Centre four for malaria channel construction. Of the 53 functional Health facilities assessed in the district, the average reporting Timeliness was $32 \%$ and Completeness at 63\% from week 44 in 2019 to week 3 in 2020. This finding shows that the district was not achieving the $80 \%$ Timeliness and $80 \%$ Completeness national target. The poor reporting situation implies that the district
\end{abstract}


may not detect an emerging Public Health Event and respond in time. Poor reporting rate was linked to knowledge gap among reporting staff in completion of the newly revised HMIS tools and lack of support supervision. The analyzed data revealed that the district had increased dysentery, measles and typhoid fever cases. The dysentery and typhoid fever cases had reached and surpassed the Alert and Action thresh hold levels however there was no reported death from these diseases. Further inquiry revealed that Typhoid fever was being diagnosed using WIDAL test as opposed to WHO recommendation of stool or blood culture. The noted typhoid fever outbreak in the district was therefore being based on wrong laboratory tests hence regarded by the researcher as speculative. Findings on malaria channel revealed a normal and expected trend of malaria in Kabarole district in 2020. Conclusion: knowledge and skills from FETP-training enabled the investigator to establish the fact that the district's surveillance system was less sensitive in detecting Public Health events for a quick response. Intensified targeted support supervision and mentorships of all health workers on reporting could help improve the districts surveillance system.

\section{Keywords}

HMIS Timeliness and Completeness, Surveillance Systems, Field Epidemiology, Malaria Chanel

\section{Introduction}

For Ministry of Health Uganda to ensure effective disease detection, reporting, investigations and good response, there is a need for a well-trained Public Health workforce in field Epidemiology. In view of this, AFENET-Uganda with CDC supported Ministry of Health Uganda to conduct a 3 months in-service Field Epidemiology Training Program (FETP)-Frontline for district surveillance teams. The FETP is designed to produce highly skilled epidemiologists who work at the ministry of health and district in the country to strengthen surveillance systems and respond to public health threats [1].

About $75 \%$ of learning under FETP arrangement occurs in the field. Trainees conduct fieldwork that increases their capacity to apply epidemiological concepts while strengthening Health System through the production of useful epidemiologic field products that provide information for decision making [2].

According to Dr. Bukirwa Hasifa, the 2019/20 FETP-Cohort was the $11^{\text {th }}$ trained cohort in Uganda that targeted about 10 districts in western Uganda and the training was conducted at Oxford Hotel Mbarara [3]. The FETP-Frontline consists of an initial 5-day workshop introducing basic epidemiology principles and importance of disease surveillance. The participants then return to their regular job sites for 5 weeks. There, they receive onsite and remote mentoring from program staff to review local surveillance data and conduct a data quality audit around a priority disease in their coverage area. All Frontline FETP par- 
ticipants create a weekly surveillance report using real-world data derived from their home districts. The FETP resident advisors and mentors then guide the participants to aggregate and analyze the data at the district level. The participants return for a second 5-day workshop to present their work and receive feedback from the staff and their peers on their projects. During the second workshop, participants learn how to conduct field investigations and how to effectively communicate results. Participants then return to the field for the second 5 -week field stage to put in practice what they have learned under the guidance of the mentors and to complete 2 of 4 possible field activities i.e. Conducting a field investigation to confirm or rule out a reportable disease, developing an expanded surveillance summary report, doing problem analysis of a surveillance quality problem and making appropriate recommendations. In the third workshop, participants present their final projects and receive a certificate of course completion cosigned by MOH Uganda and CDC representatives [4].

\section{Background}

This article presents detailed account of Field Epidemiology Training Program (FETP) activities done by the investigator during field placement two. The aim of Uganda FETP is to prepare the frontline participant to be a competent field epidemiology officer capable of detecting early and instituting a correct response to any Public Health issues in the population [1]. This concurs with Centre for Disease Control and Prevention (CDC) goal of supporting several Government ministries of Health to produce highly skilled epidemiologists who can work at ministry of health in the country to strengthen surveillance systems and respond to public health threats [4].

In 2015, the US centers for Disease Control and Prevention launched FETP frontline, a 3 months field training programme targeting $\mathrm{MOH}$ staff and District staff in countries. This was aimed at enhancing local Public Health capacity so as to briskly detect, respond and contain Public Health Emergencies at the source. [5]. Among other requirements to complete the FETP, the Uganda FETP $11^{\text {th }}$ cohort members were required to: Analyze the cause of a quality problem, use the recommended and known WHO, MOH, and IDSR Alert threshold and Action threshold and do trend analysis of frequently reported epidemic prone diseases and finally extract data on malaria cases for five years and use it to construct malaria normal channel for the district. The researcher in this investigation was a FETP-graduate of the $11^{\text {th }}$ cohort in 2020 from Uganda, applying the knowledge and skills acquired to strengthen the district surveillance system.

\section{Methods}

\subsection{Study Design}

An institutional based, epidemiological investigation with cross-sectional design was conducted at district level and Health Centre $1 \mathrm{~V}$ in Kabarole district from $15^{\text {th }}$ December 2019-March 2020. Data review was done at district health office 
and at the Health Centre IV using DHIS-2 eReports.

\subsection{Study Area}

The assessment was done in Kabarole district located in western Uganda at a road Distance of $300 \mathrm{~km}$ from Kampala city. Kabarole is comprised of 2 counties (HSDs); Burahya and Fort Portal Municipality.

It consists of 4 town councils, 3 divisions, 11 sub-counties, 59 parishes and 523 villages. It has a population of 328,500 people with an annual growth rate of $2.3 \%[6]$.

\subsection{Sample Size}

In this assessment all the 53 Functional Health facilities in Kabarole district were included and the electronic weekly reports (HMIS 033b) retrieved in DHIS-2 system. For the malaria channel, Construction, Kataraka Health Centre 1V was purposively selected on ground that it had consistently reported during the assessment period with Timeliness at $92 \%$ and $100 \%$ completeness.

\section{Data Collection Tools}

In this study, pre-designed data collection checklist was used to guide in data extraction from DHIS 2 and from the Health Centre data base to get the number of HMIS 033 weekly reports submitted and the weekly malaria cases seen.

\subsection{Data Collection Technique}

Data Quality Audit (DQA) was done to identify all issues around data collection, reporting, analysis and use of data. From the DQA findings, SWOT analysis was done and the lead data quality problems (weaknesses) identified. Participatory approach through a brainstorming session to analyze the identified problem and the underlying causes was then done. Raw Data was retrieved from the electronically reported HMIS weekly reports in DHIS-2 for the expanded surveillance activity and also for the malaria channel construction. Face to face mentorships and guidance to Health facility staff at Kataraka HC IV was also done during the joint completion of the malaria channel construction.

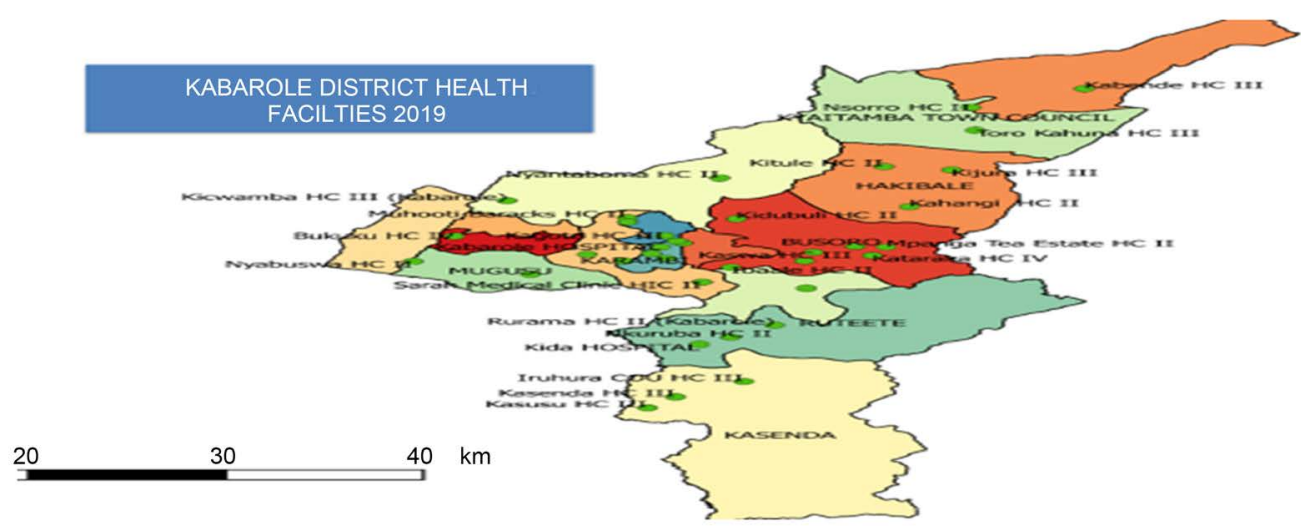

Figure 1. Geographical distribution of health facilities assessed. 


\subsection{Ethical Considerations}

This assessment was focused on electronic data in DHIS-2. The investigation was determined to be non-human subject research according to Uganda's research guidelines [7]. Authorization of the study was obtained from the District health officer and the Health officer in-charge of Kataraka Health Centre IV. The Health facility staffs who participated in data use in construction of malaria channel were provided with information that their participation was voluntary and the activity was meant to build their capacity in malaria epidemic detection and their refusal would not lead to any consequences.

\section{Results: Expanded Surveillance Activity}

Table 1 presents the weekly reporting trend for HMIS 033b in Kabarole district. From week 44 in 2019 to week 3 in 2020, 53 weekly reports from all the Health facilities were expected. Raw 3 of the table shows reports received every week and raw 4 shows the reports received on time. Weekly trend on timeliness and completeness is also shown.

The trend seen in Figure 2 shows, there is no single month when all the 53 expected reports were received translating to an average completeness of $63 \%$ against a target of $80 \%$. On timeliness, the situation appears worse, the few reports that were receive weekly most of them were late depicting an average weekly reporting timeless of $32 \%$ against a target of $80 \%$.

Figure 3 presents Timeliness and Completeness of the weekly reporting for HMIS 033b in Kabarole district against a national target of $80 \%$. The trend seen above shows that from week 44 in 2019 to week 3 in 2020, performance in both indicators i.e. timeliness and completeness was below target of $80 \%$. On average completeness was at $63 \%$ while timeliness was at $32 \%$ against a target of $80 \%$.

Table 2 shows the weekly reporting status in the12 selected Health Facilities. It is noted that Kataraka HC 1V, Katojo HC 111 and KasendaHC 111 consistently reported on time achieving and surpassing the $80 \%$ target while Kijura, Mucwa and Muhoti HCs continued to deteriorate during the reporting indicated.

Table 1. Kabarole district hmiso33b weekly reporting status (WK $44^{2019}$ - WK $3^{2020}$ ).

\begin{tabular}{lcccccccccccc}
\hline & Wk & Wk & Wk & Wk & Wk & Wk & Wk & Wk & Wk & Wk & Wk & Wk \\
& 44 & 45 & 46 & 47 & 48 & 49 & 50 & 51 & 52 & 01 & 02 & 03 \\
\hline $\begin{array}{l}\text { Total reports expected in } \\
\text { week }\end{array}$ & 53 & 53 & 53 & 53 & 53 & 53 & 53 & 53 & 53 & 53 & 53 & 53 \\
$\begin{array}{l}\text { Total reports received in } \\
\text { week }\end{array}$ & 38 & 37 & 43 & 44 & 37 & 42 & 44 & 28 & 26 & 21 & 34 & 30 \\
$\begin{array}{l}\text { Total reports on time by } \\
\text { week }\end{array}$ & 17 & 22 & 19 & 22 & 15 & 23 & 19 & 18 & 15 & 12 & 12 & 9 \\
$\begin{array}{l}\text { \% completeness } \\
\text { \% Timeliness }\end{array}$ & 72 & 70 & 81 & 83 & 70 & 79 & 83 & 53 & 49 & 40 & 64 & 57 \\
\hline & 32 & 42 & 36 & 42 & 28 & 43 & 36 & 34 & 28 & 23 & 23 & 17 \\
\hline
\end{tabular}


Table 2. Kabarole weekly reporting pattern of the 12 selected H/Fs wk 44-2019-wk 3 (2020).

\begin{tabular}{|c|c|c|c|c|c|c|c|c|c|c|c|c|c|}
\hline Health Facility & $\begin{array}{l}\text { W44 } \\
2019\end{array}$ & $\begin{array}{l}\text { W45 } \\
2019\end{array}$ & $\begin{array}{l}\text { W46 } \\
2019\end{array}$ & $\begin{array}{l}\text { W47 } \\
2019\end{array}$ & $\begin{array}{l}\text { W48 } \\
2019\end{array}$ & $\begin{array}{l}\text { W49 } \\
2019\end{array}$ & $\begin{array}{l}\text { W50 } \\
2019\end{array}$ & $\begin{array}{l}\text { W51 } \\
2019\end{array}$ & $\begin{array}{l}\text { W52 } \\
2019\end{array}$ & W1 2020W & 22020 & $\begin{array}{l}\text { wk } 3 \\
2020\end{array}$ & $\begin{array}{c}\% \text { cumulative } \\
\text { Timeliness }\end{array}$ \\
\hline Bukuku HC IV & $\mathrm{T}$ & $\mathrm{T}$ & $\mathbf{L}$ & $\mathrm{T}$ & $\mathbf{L}$ & $\mathrm{T}$ & L & NR & NR & NR & NR & $\mathrm{T}$ & $63 \%$ \\
\hline F/Portal Police HC 2 & NR & $\mathrm{T}$ & $\mathrm{T}$ & $\mathrm{T}$ & $\mathrm{T}$ & $\mathrm{T}$ & $\mathrm{T}$ & $\mathrm{T}$ & NR & $\mathbf{L}$ & $\mathbf{L}$ & $\mathrm{L}$ & $64 \%$ \\
\hline Kataraka HC IV & $\mathrm{T}$ & $\mathrm{T}$ & $\mathrm{T}$ & $\mathrm{T}$ & $\mathrm{T}$ & $\mathrm{T}$ & $\mathrm{T}$ & $\mathrm{T}$ & $\mathbf{L}$ & $\mathrm{T}$ & $\mathrm{T}$ & $\mathrm{T}$ & $92 \%$ \\
\hline Katojo HC3 & $\mathrm{T}$ & $\mathrm{T}$ & $\mathrm{T}$ & $\mathrm{T}$ & $\mathrm{T}$ & $\mathrm{T}$ & $\mathrm{T}$ & $\mathrm{T}$ & $\mathbf{L}$ & NR & $\mathrm{T}$ & $\mathrm{L}$ & $82 \%$ \\
\hline Kicwamba HC III & $\mathrm{L}$ & NR & $\mathbf{L}$ & $\mathbf{L}$ & $\mathbf{L}$ & $\mathrm{L}$ & $\mathrm{T}$ & NR & $\mathbf{L}$ & NR & $\mathrm{T}$ & NR & $25 \%$ \\
\hline Kijura HC 3 & $\mathrm{~L}$ & L & $\mathbf{L}$ & L & NR & $\mathbf{L}$ & $\mathbf{L}$ & NR & NR & $\mathrm{L}$ & $\mathbf{L}$ & $\mathrm{T}$ & $11 \%$ \\
\hline Mucwa HC III & $\mathbf{L}$ & NR & L & L & NR & $\mathrm{L}$ & $\mathrm{T}$ & NR & NR & $\mathrm{T}$ & NR & NR & $14 \%$ \\
\hline Mugusu HC3 & $\mathrm{T}$ & $\mathrm{L}$ & L & $\mathrm{T}$ & $\mathrm{L}$ & L & $\mathbf{L}$ & $\mathrm{L}$ & NR & NR & NR & $\mathrm{L}$ & $22 \%$ \\
\hline Muhooti HC 11 & $\mathrm{~L}$ & NR & NR & $\mathrm{L}$ & $\mathbf{L}$ & $\mathrm{L}$ & $\mathbf{L}$ & $\mathrm{T}$ & NR & NR & NR & NR & $17 \%$ \\
\hline Kabende HC3 & $\mathrm{L}$ & $\mathrm{L}$ & $\mathbf{L}$ & $\mathrm{T}$ & $\mathbf{L}$ & NR & L & NR & $\mathrm{T}$ & NR & NR & $\mathrm{L}$ & $25 \%$ \\
\hline Kasenda $\mathrm{HC} 3$ & $\mathrm{~T}$ & $\mathrm{~T}$ & $\mathrm{~T}$ & $\mathrm{~T}$ & $\mathrm{~T}$ & $\mathrm{~T}$ & $\mathrm{~T}$ & L & NR & $\mathrm{T}$ & $\mathbf{L}$ & NR & $89 \%$ \\
\hline Rutete HC3 & $\mathrm{T}$ & $\mathrm{L}$ & $\mathrm{T}$ & $\mathrm{L}$ & $\mathrm{L}$ & $\mathrm{T}$ & $\mathrm{T}$ & $\mathrm{T}$ & $\mathrm{T}$ & NR & $\mathrm{T}$ & $\mathrm{L}$ & $55 \%$ \\
\hline
\end{tabular}

KEY: $\mathrm{T}=$ Timey: $\mathrm{L}=$ Late Report: $\mathrm{NR}=$ No report.

$\square \mathrm{T}=$ or $>50 \%$ but $<80 \%$;

$\mathrm{T}=0 \mathrm{R}>80 \%$;

$\mathrm{T} \leq 50 \%$.

Kabarole weekly reporting status HMIS 033B in WK 44 (2019)- WK 3 (2020)

Total expected in wk Total reported in wk Total reports on time

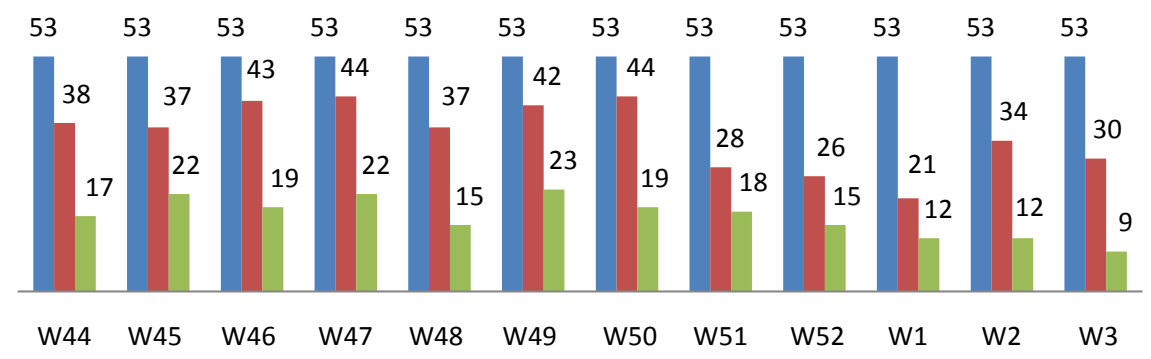

Figure 2. Kabarole HMIS 033b expected reports, received \& reports on time.

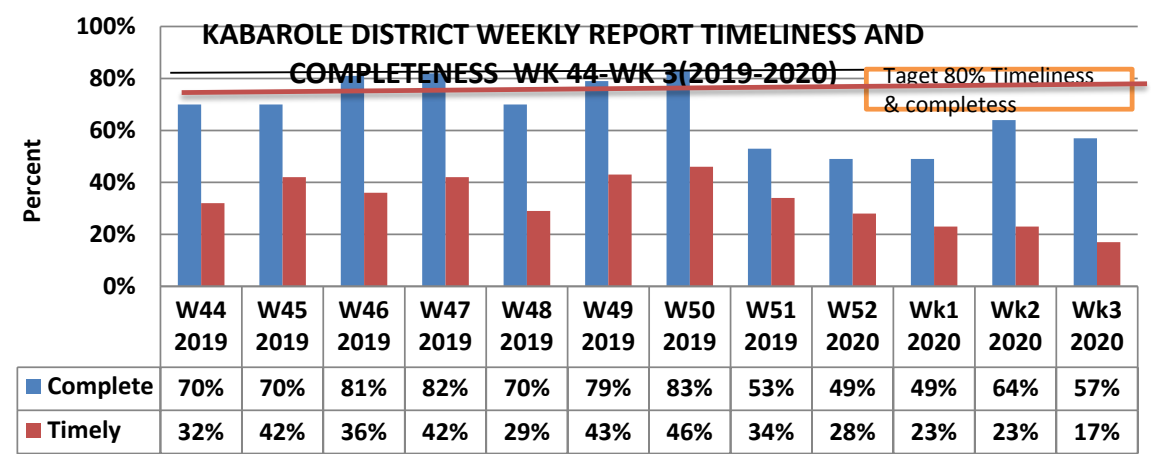

Figure 3. Kabarole HMIS 033b timelines and completeness of reporting.

Table 3 displays data on the 3 frequently reported epidemic prone diseases during the assessment period. Cases and death from Typhoid fever, Measles and Dysentery are tabulated as the most reported conditions. 
Table 3. Kabarole district epidemic prone diseases in wk 442019 - wk 3, 2020. Cases and deaths by week.

\begin{tabular}{|c|c|c|c|c|c|c|c|c|c|c|c|c|}
\hline Disease & W44. & W45 & W46 & W47 & W48 & W49 & W50 & W51 & W52 & W1 & W2 & W3 \\
\hline $\begin{array}{l}\text { Dysentery } \\
\text { Cases }\end{array}$ & 12 & 8 & 3 & 5 & 1 & 3 & 0 & 1 & 3 & 0 & 3 & 1 \\
\hline $\begin{array}{l}\text { Dysentery } \\
\text { Deaths }\end{array}$ & 0 & 0 & 0 & 0 & 0 & 0 & 0 & 0 & 0 & 0 & 0 & 0 \\
\hline $\begin{array}{l}\text { Measles } \\
\text { Cases }\end{array}$ & 1 & 0 & 1 & 0 & 0 & 1 & 0 & 0 & 1 & 0 & 0 & 0 \\
\hline $\begin{array}{c}\text { Measles } \\
\text { Deaths }\end{array}$ & & 0 & 0 & 0 & 0 & 0 & 0 & 0 & & 0 & 0 & 0 \\
\hline $\begin{array}{l}\text { Typhoid F } \\
\text { Cases }\end{array}$ & 15 & 13 & 25 & 29 & 20 & 17 & 15 & 2 & 8 & 12 & 12 & 0 \\
\hline Typhoid F D & 0 & 0 & 0 & 0 & 0 & 0 & 0 & 0 & 0 & 0 & 0 & 0 \\
\hline
\end{tabular}

Figure 4 shows the trend of most frequently reported diseases as dysentery, measles and typhoid fever. The number of cases for all the 3 diseases increased in week 44 and week 47, from week 51 to week 3 , cases for the three conditions were consistently in a low \& downward trend.

Figure 5 shows that the alert threshold for typhoid fever was reached and surpassed in week 46 and the Action threshold also surpassed in the same week up to week 48. Alert thresh hold was considered as having a notable increase in typhoid fever cases and the Action threshold as having 20 suspected cases of typhoid seen in the district as by standard WHO guidelines [8].

From Figure 6, it's noted that district was in Alert phase for measles in week $44,46,49 \& 52$. By experiencing one suspected case of measles, the district is in alert phase.

Figure 7 shows the district reached Alert and Action threshold for dysentery for 2 consecutive weeks (wk 44 and wk 45). Alert threshold is when there is un usual clustering of 5 or more cases of Bacillary dysentery from a parish in a week, Action threshold is when the cases are consistently above Alert threshold for 2 weeks. From week 46, cases dropped and remained below alert phase up to week 3 in 2020.

Field Activity 2: Analysis of a Surveillance Quality Problem

During phase 1 of the investigation, Data Quality Audit (DQA) was done in 12 selected health facilities in the district. Selection criteria of the 12 health facilities reflected in Table 2 was based on; hard to reach health facility, high volume and low surveillance capacities. Findings of the DQA were categorised into Strength, Weaknesses, Opportunities and Threats. The most crosscutting weakness found was Poor HMIS reporting manifested by incomplete and late reporting. This situation prompted the investigator to do route cause analysis using Fish bone tool so as to have a deeper understanding of the problem and recommend practical interventions to correct the situation (Figure $8 \&$ Table 4). 
Weekly trend of 3 priority IRDs(Dysentery, Measles and Typhoid fever)

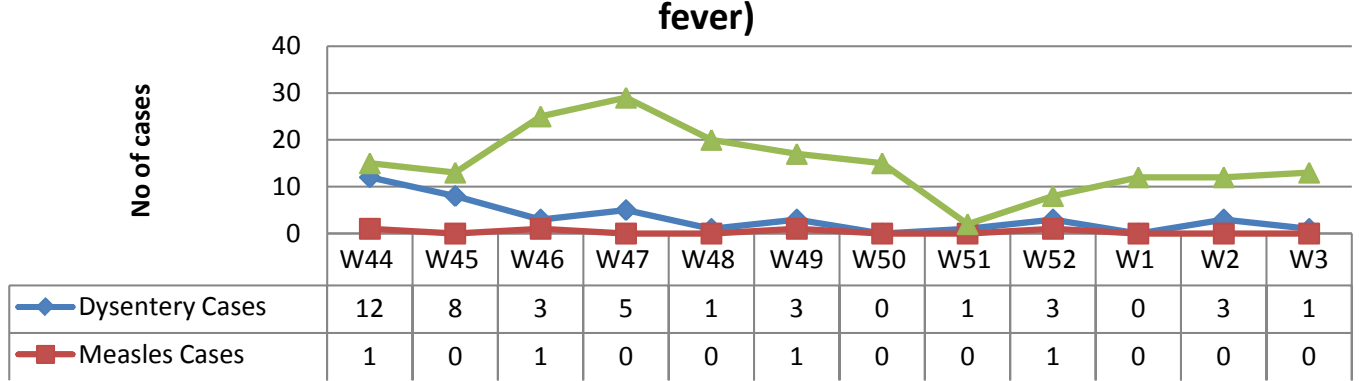

Figure 4. Trend of dysentery, measles \& typhoid fever in Kabarole wk 44 (2019) - wk 3 (2020).

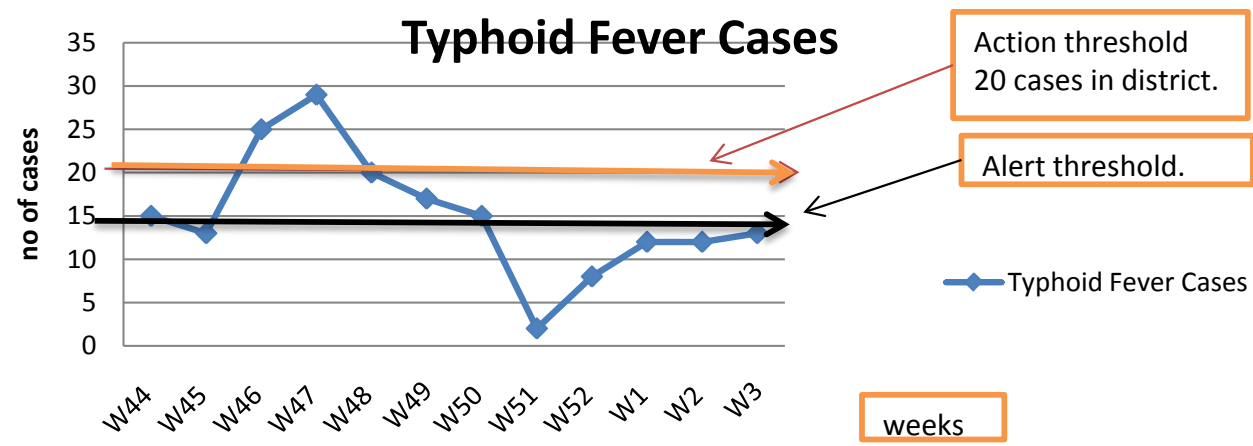

Figure 5. Weekly trend of typhoid fever cases, the alert and action threshold.

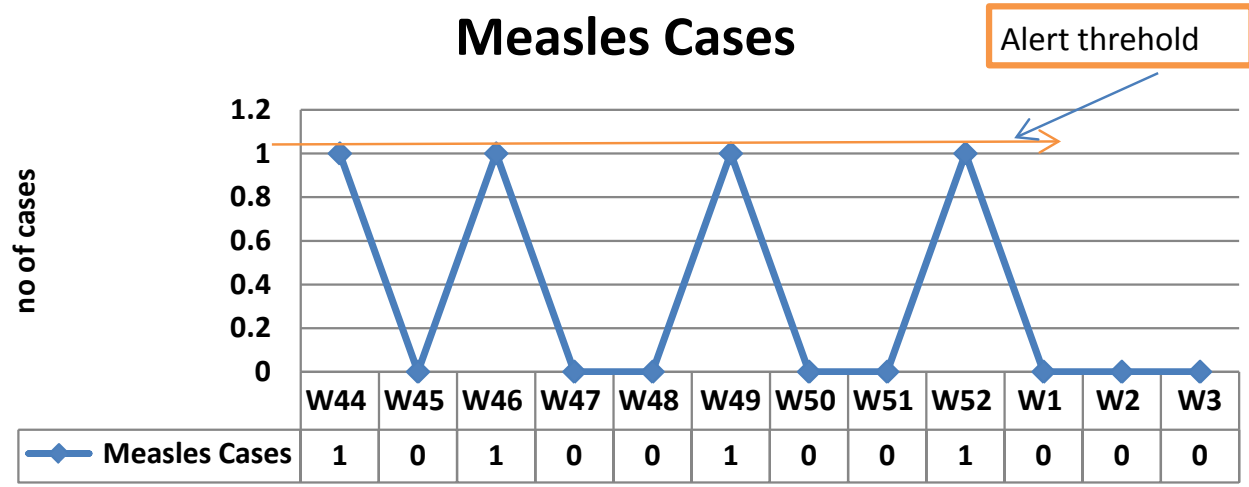

Figure 6. Measles cases seen in week 44(2019) - wk 3 (2020).

\section{Dysentery Cases}

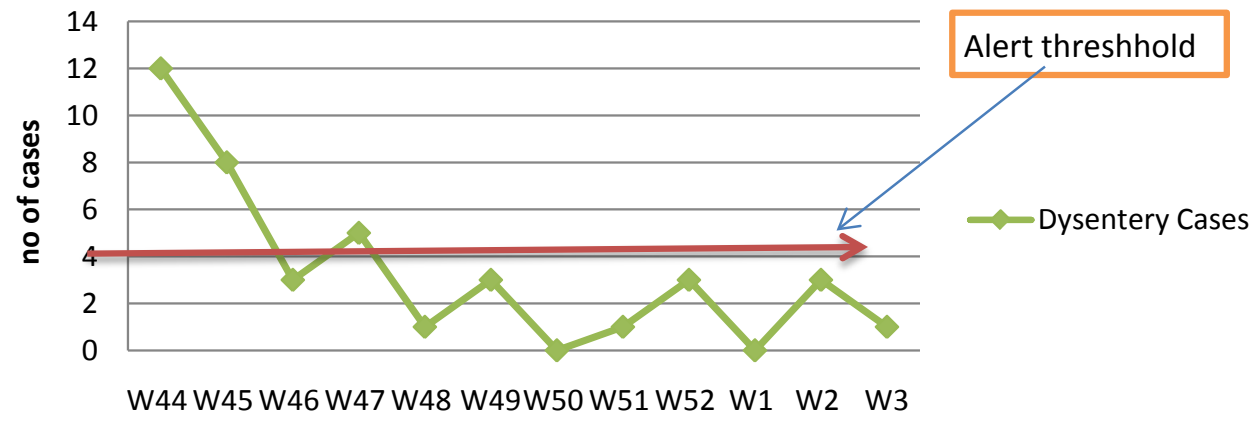

Figure 7. Dysentery cases seen in week 44(2019) - wk 3 (2020). 


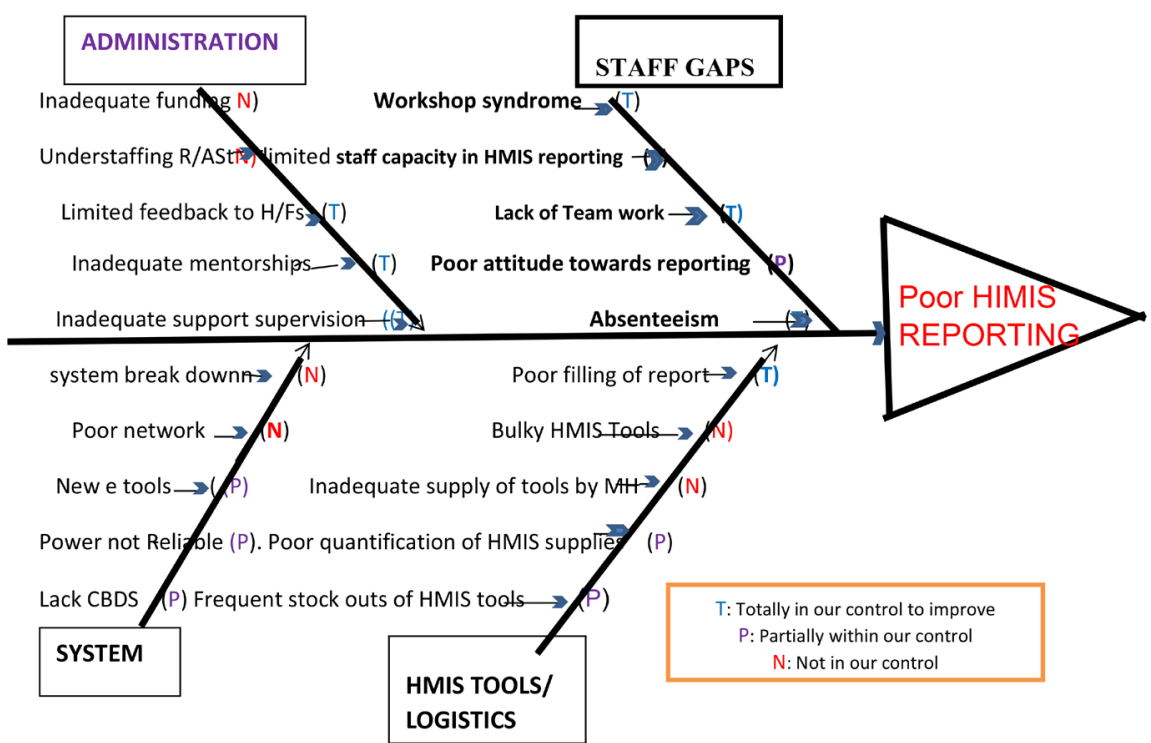

Figure 8. Poor HMIS reporting in Kabarole causes and route causes-Fish Bone tool.

Table 4. Problem analysis worksheet-summarising the fish bone findings.

SURVEILLANCE QUALITY PROBLEM
POOR HMIS REPORTING IN KABAROLE HEALTH FACILITIES
UNDERLYING CAUSES
1) LIMITED HEALTH WORKERS CAPACITY IN HMIS REPORTING (STAFF GAPS)
2) HMIS TOOLS/LOGISTICS CONCERNS
3) ADMINISTRATIVE ISSUES ( INADEQUATE SUPPORT SUPERVISION)
4) HMIS REPORTING SUPPORTING SYSTEM CONCERNS
CRITICAL CAUSE (based On T and P)
IMPROVEMENT RECOMMENDATIONS
INADEQUATE SUPPORT SUPERVISION AND MENTORSHIPS OF HEALTH
1) DHT TO STRENTHEN TARGETED SUPPORT SUPERVISION TO HEALTH
FACILITIES ON HMIS REPORTING
2) DHT CONDUCT ON JOB MENTORSHIPS ON USE OF REVISED HMIS TOOLS
3) MOH \& BIOSTAT PROVIDE REGULAR FEEDBACK ON HMIS REPORTING
PERFORMANCE TO REPORTING H/FS
4) WORK WITH HEALTH FACILITY INCHARGES TO ADREES ABSENTISM
BY REWARDS AND SANCTIONS APPROACH.
5) ORGANISE AND HOLD DATA REVIEW MEETINGS TARGETING ALL
STAKEHOLDERS ON QUARTERLY BASIS.

Field Activity 3: Construction of Malaria channel; -The Alert and Action thresh hold.

The available IDSR guidelines outline disease specific thresholds for most priority diseases and Public Health conditions and events. A thresh hold is a pointer to a health worker that once a disease condition reaches the specified thresh hold level, then some action should be taken. According to MOH Uganda IDSR guidelines [9], the two types of threshold that should be considered are: -Alert Threshold and Action thresh hold. Alert threshold calls for immediate 
investigation of the suspected disease while Action threshold calls for immediate response. Whereas most diseases/events have distinct threshold numbers, malaria being a highly endemic disease, the thresh holds are determined using malaria channel based on malaria weekly reported for 52 weeks in a year. The mean for each week in the five years is determined for all the weeks and then a mean plus 1SD for each week in 5 years is also computed. The two variables are then plotted to form the normal channel. Malaria cases for the year under observations are then plotted in respect to the channel. If the currently observed cases fall outside the upper limit of the channel, then a malaria Action threshold is reached. This calls for immediate reporting to higher levels and for quick investigations [8].

Kataraka Health centre $1 \mathrm{~V}$ in Kabarole was selected for this activity given the fact that this Health facility had attained the Timeliness and completeness indicators at $92 \%$ and $98 \%$. Table 2 attests to this timely, complete and reliable data for action. Health facility surveillance focal person and the HMIS focal persons were involved and mentored on malaria channel construction using the very data they had compiled as shown in Table 5.

Table 5 shows the malaria cases by week for 5 years. The mean for 5 years, Standard deviation, mean plus one standard deviation and the observed malaria cases in 2020.

Figure 9 shows that, the number of malaria cases in year 2020 from week 1 to week 7 fall between the upper and the lower limits of a normal malaria channel. This reflects a normal and expected trend of malaria at Kataraka HCIV in Kabarole district. If similar trends are seen in other Health Facilities, one can confidently say that the district is not under malaria threat as of week 7 in 2020.

\section{Discussion, Conclusions and Recommendations}

The objectives of the assessment were, to do expanded surveillance analysis of weekly reporting in all district Health facilities [Figure 1] focusing on timeliness and completeness, identify the most frequently reported epidemic prone

Table 5. Kataraka HC 1V Weekly malaria cases for the period 2015-2019. Mean SD and Mean + 1 SD.

\begin{tabular}{|c|c|c|c|c|c|c|c|c|c|c|c|c|c|c|c|c|c|c|c|c|}
\hline Wk no & Wk01 & W2 & W3 & W4 & W5 & W6 & W7 & W8 & w9 & wk10 & wk11 & wk12 & wk13 & wk14 & wk15 & wk16 & wk17 & wk18 & wk19 & wk20 \\
\hline 2015 & 3 & 5 & 9 & 9 & 9 & 11 & 7 & 3 & 4 & 5 & 9 & 6 & 8 & 8 & 9 & 5 & 10 & 6 & 9 & 11 \\
\hline 2016 & 3 & 5 & 7 & 6 & 10 & 12 & 8 & 9 & 5 & 9 & 10 & 11 & 9 & 5 & 11 & 6 & 7 & 7 & 2 & 8 \\
\hline 2017 & 11 & 8 & 10 & 9 & 8 & 6 & 3 & 12 & 6 & 3 & 9 & 6 & 7 & 10 & 8 & 9 & 6 & 5 & 8 & 2 \\
\hline 2018 & 4 & 4 & 7 & 5 & 2 & 2 & 5 & 4 & 1 & 1 & 3 & 1 & 2 & 0 & 3 & 0 & 3 & 2 & 3 & 7 \\
\hline 2019 & 5 & 24 & 8 & 8 & 6 & 6 & 3 & 5 & 3 & 5 & 7 & 2 & 12 & 9 & 10 & 5 & 8 & 3 & 11 & 3 \\
\hline Average & 5 & 9.2 & 8 & 7 & 7 & 7 & 5 & 7 & 4 & 5 & 8 & 5 & 8 & 6 & 8 & 5 & 7 & 5 & 7 & 6.2 \\
\hline STDEV & 3 & 8.4 & 1 & 2 & 3 & 4 & 2 & 4 & 2 & 3 & 3 & 4 & 4 & 4 & 3 & 3 & 3 & 2 & 4 & 3.7 \\
\hline$x+1$ STD & 9 & 18 & 10 & 9 & 10 & 12 & 8 & 10 & 6 & 8 & 10 & 9 & 11 & 10 & 11 & 8 & 9 & 7 & 11 & 9.9 \\
\hline Yr 2020 & 3 & 3 & 8 & 9 & 5 & 5 & 6 & & & & & & & & & & & & & \\
\hline
\end{tabular}




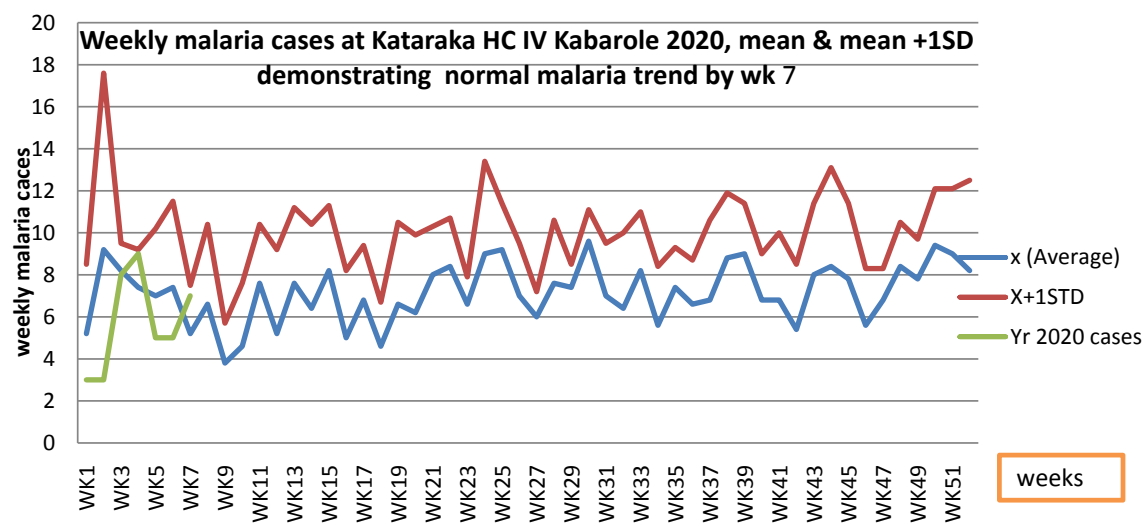

Figure 9. Weekly malaria cases at Kataraka HC IV Kabarole district by week 7 in 2020 demonstrating a normal malaria trend.

diseases in 12 weeks (wk 44 - wk 3) and do trend analysis, establish the priority data quality problem in the district and establish underlying causes as well as supporting staff in one Health facility on construction of a malaria channel.

Analysis of weekly reporting in the 53 Health facilities in Kabarole showed that from week 44 in 2019 to week 3 in 2020 the average reporting timeliness was $32 \%$ and completeness at $63 \%$. The two indicators are far much below the $\mathrm{MOH}$ set target of $80 \%$ [9]. Contrary to this finding, other studies done in Uganda and elsewhere [10] [11] have shown improvements in completeness and timeliness of reporting. The Low reporting rate implies that the district may not detect an epidemic threat of public health nature and institute a timely action. Late and low reporting could be attributed to knowledge gap among reporting staff on the importance of timely reporting. Previous studies [12] conducted in other settings have also linked poor health workers performance in surveillance to lack of adequately trained personnel. Interaction with Records Assistants during the DQA established the problem of newly introduced bulky HMIS reporting formats. This concurs with the report earlier on presented by the district Biostatistician [13] that records Assistants were oriented in the newly introduced HMIS forms for only 2 days instead of at least 5 days. The knowledge gap on use of revised tools could be overcome by the district soliciting for extra financial resources to train and mentor the HMIS reporting staffs.

Findings in [Table 3 and Figure 4] further revealed that the frequently reported epidemic prone diseases were dysentery, measles and typhoid fever. No death was however noted for any of the mentioned diseases. Both dysentery and typhoid fever had reached the epidemic proportion levels. WHO IDSR guidelines show that 20 suspected cases of typhoid fever in a district catchment imply that Action threshold is reached. The trend analysis shows [Figure 5] in wk 46, 47 and 48 there were over 20 cases seen, meaning that the district had been with un-noticed out-break of typhoid fever. Further inquiry revealed that diagnosis was being done using WIDAl test which is just a screening test. WHO recommends Blood or stool Culture as confirmatory diagnostic test for salmonella spp. 
Other studies have identified inadequate laboratory capacity for disease confirmation and limited availability of laboratory supplies as limiting factors in surveillance [10]. This literally means that the typhoid fever outbreak shown by data is not real and the consequences of such a practice are insurmountable ranging from irrational use of Antibiotics in treating none existing condition, economic drain of patients in purchasing expensive antibiotics to creating resistance to local antibiotics.

SWOT analysis revealed that Poor HMIS reporting was the main surveillance quality problem. Route Cause Analysis [Figure 8] revealed that Administrative Issues like Inadequate Support Supervision by the district Health Team was the underpinning factor. Irregular support supervision for IDSR activities has previously been pointed out as a significant challenge affecting surveillance in other studies [14]. Through regular support supervision, health systems challenges can be identified and fixed at early stage.

Malaria epidemics are usually detected using malaria channel. WHO recommends 2 approaches in constructing the malaria channel depending on the Health facility level? [15] National, District level and Health Sub District level. Malaria cases by week for the previous 5 years are organised. Using EXEL the mean for 5 years is determined and tabulated then the mean +ISTEDV. In lower level health Facilities, The mean and median $\left(75^{\text {th }}\right.$ percentile is used to construct the channel. Taking all this into account, malaria channel for Kataraka HC IV was constructed. When malaria cases for 2020 were plotted, the channel revealed a normal and expected trend of malaria at Kataraka HCIV and therefor Kabarole district. For this to be more meaningful all reporting health facilities should construct their respective malaria channels.

\section{Conclusion}

Kabarole district HMIS weekly reporting Timeliness at $32 \%$ and completeness at $63 \%$ are far much below the $\mathrm{MOH}$ and WHO target of $80 \%$. This shows the district surveillance system is less sensitive to detect serious epidemic prone diseases. Health facilities diagnose typhoid fever by Widal test instead of doing blood or stool culture and this creates theoretical out breaks. Inadequate support supervision and mentorships of health workforce on the newly revised data reporting tools were identified as the main underlying cause of the poor HMIS reporting indicators.

\section{Recommendations}

The district surveillance team should intensify targeted support supervision and mentorships of all health workers on reporting. The district surveillance officer should intensify awareness creation on use of blood culture or stool culture for laboratory diagnosis of enteric fevers.

The District surveillance officer should roll out mentorships of health workers on construction and use of malaria channel. 


\section{Limitations of the Study}

Dealing with data helped the researcher to understand the trends HMIS reporting and the reported disease but the researcher could not understand Health facility specific underlying causes for the poor reporting.

The normal trend of malaria in the district was based on data of one Health Centre IV. A clear picture would have been got if this was done in all 53 health Facilities.

\section{Acknowledgements}

I acknowledge the technical and financial support by AFENET and CDC that enabled us complete the project. The Volunteer Biostatistician Mr. Musoni Wilson who helped us accesses the electronic data in DHIS2 is also acknowledged.

\section{Conflicts of Interest}

There are no competing interests.

\section{References}

[1] (2017) Field Epidemiology Training Program Frontline FETP Uganda. Workshop 1 Participant Guide.

[2] André, A., Lopez, A., Perkins, S., Lambert, S., Chace, L., Noudeke, N. and Pedalino, B. (2017) Frontline Field Epidemiology Training Programs as a Strategy to Improve Disease Surveillance and Response. Emerging Infectious Diseases, 23, S166-S173. https://doi.org/10.3201/eid2313.170803

[3] Hasifa, B. (2020) Uganda FETP Field Trainer and Supervisor 2019/2020.

[4] Mukanga, D., Namusisi, O., Gitta, S.N., Pariyo, G., Tshimanga, M., Weaver, A., et al. (2010) Field Epidemiology Training Programs in Africa-Where Are the Graduates? Human Resources for Health, 8, 18. https://doi.org/10.1186/1478-4491-8-18

[5] (2015) US Department of Health and Human Services (CDC), Centre for Global Health, Division of Global Health Protection, and Field Epidemiology Training Branch.

[6] Uganda Bureau of Statistics (Web). Kabarole District Population 2020.

[7] Uganda National Council for Science and Technology. National Guidelines for Research Involving Humans as Research Participants. https://uncst.go.ug/guidelines-and-forms

[8] WHO (2012) Case Definitions and Epidemic Thresholds for Intergraded Disease Surveillance and Response.

[9] MOH (2012) National Technical Guidelines for Integrated Disease Surveillance and Response.

[10] Lukwago, L., Nanyunja, M., Ndayimirije, N., Wamala, J., Malimbo, M., Mbabazi, W., et al. (2012) The Implementation of Integrated Disease Surveillance and Response in Uganda: A Review of Progress and Challenges between 2001 and 2007. Health Policy Plan, 28, 30-40. https://doi.org/10.1093/heapol/czs022

[11] Adokiya, M.N., Awoonor-Williams, J.K., Beiersmann, C. and Müller, O. (2015) The Integrated Disease Surveillance and Response System in Northern Ghana: Challenges to the Core and Support Functions. BMC Health Services Research, 15, 288. 
https://doi.org/10.1186/s12913-015-0960-7

[12] Sow, I., Alemu, W., Nanyunja, M., Duale, S., Perry, H.N. and Gaturuku, P. (2010) Trained District Health Personnel and the Performance of Integrated Disease Surveillance in the WHO African Region. East African Journal of Public Health, 7, 16-19. https://doi.org/10.4314/eajph.v7i1.64671

[13] Byaruhanga, C. (2020) Kabarole Biostatistician Report on the 2 Days of Training of Records Assistants in Revised HMIS Tools.

[14] Wamala, J.F., Okot, C., Makumbi, L., Kisakye, A., Nanyunja, M., et al. (2010) Assessment of Core Capacities for the International Health Regulations (IHR) [2005] Uganda, 2009. BMC Public Health, 10, S9. https://doi.org/10.1186/1471-2458-10-S1-S9

[15] WHO-AFRO and CDC (2012) Integrated Disease Surveillance and Response (IDSR). District Level Training Resource.

\section{Abbreviations}

DHIS-2: District Health Information Software Version-2

DQA; Data Quality Audit

CDC: Centre for Disease Control and Prevention.

$M O H$; Ministry Of Health

FETP-Frontline.: Field Epidemiology Training program

DHT: District Health Team

SWOT: Strength Weakness Opportunities Threats.

IDSR: Intergraded Disease Surveillance and Response

WHO: World Health Organization.

1SD: One Standard Deviation 